\title{
Design and Implementation of a Compact High-Throughput Echelle Spectrometer Using Off-the-Shelf Off-Axis Parabolic Mirrors for Analysis of Biological Samples by LIBS
}

\author{
Hamed Abbasi $^{* a}$, Georg Rauter ${ }^{\mathrm{b}}$, Raphael Guzman ${ }^{\mathrm{c}}$, Philippe C. Cattin ${ }^{\mathrm{d}}$, Azhar Zam ${ }^{\dagger a}$ \\ a Biomedical Laser and Optics Group (BLOG), Department of Biomedical Engineering, University of Basel, \\ 4123 Allschwil, Switzerland \\ ${ }^{\mathrm{b}}$ Bio-Inspired RObots for MEDicine-Lab. (BIROMED-Lab), Department of Biomedical Engineering, \\ University of Basel, 4123 Allschwil, Switzerland \\ ${ }^{\mathrm{c}}$ Department of Neurosurgery, University Hospital Basel, 4056 Basel, Switzerland \\ ${ }^{\mathrm{d}}$ Center for medical Image Analysis and Navigation (CIAN), Department of Biomedical Engineering, \\ University of Basel, 4123 Allschwil, Switzerland
}

*hamed.abbasi@unibas.ch; hamedabbasi@gmail.com; phone+41 (0)61 2075461

†azhar.zam@unibas.ch; phone+41 (0)61 20754 60; http://blog.dbe.unibas.ch/

\begin{abstract}
This work presents the development of an Echelle spectrometer that is optimized for the characterization of laser-driven plasma emission of biological samples for application in smart laser surgery systems. Despite the compact (portable) and cost-efficient design of the developed spectrometer, it allows analyzing the spectrum of a plasma emitted from bone, and its surrounding soft tissues (bone marrow, muscle, and fat) in nearly the same way as a full-sized Echelle spectrometer as used in commercial laser-induced breakdown spectroscopy (LIBS) systems. Most of the commercially available Echelle spectrometers on the market use a long focal length on-axis mirror to have a reasonable F number (which defines the optical throughput of the system) and low aberration. While a long focal length requires less tilting of the mirror than a shorter focal length (the higher the tilt angle, the higher the aberration), a long focal length increases the system size and decreases sensitivity (i.e., less optical throughput).

In this work, a parabolic $90^{\circ}$ off-axis mirror with a focal length of $152.4 \mathrm{~mm}$ and a diameter of $50.8 \mathrm{~mm}$, which leads to an F-number of 3, has been used. This low F-number provides a high optical throughput compared to other similar commercial Echelle spectrometers with F-numbers of 10 or more [1-5]. Since most of the important peaks in biological tissue are in the interval of 240 to $840 \mathrm{~nm}$ [6], the design was done by using off-the-shelf aluminum mirrors with a UV-enhanced coating for both collimating and focusing purposes to cover this range with sub-Angstrom resolution. Both collimating and focusing mirrors were chosen with the same radius of curvature and declination angle (opposite direction) to cancel the coma. In this antiparallel configuration, the second parabolic mirror largely eliminates the aberrations from the first one. Moreover, we positioned the Echelle grating under the condition of quasi-Littrow design to have high diffraction efficiency with an off-axis angle in the horizontal plane. A ruled reflection grating with dispersion perpendicular to that of the Echelle grating was utilized as a cross dispenser (order separator) after the Echelle grating to distinguish the overlapping diffraction harmonics. The spectrometer has been connected to a gated ICCD to measure time-resolved spectra. The developed spectrometer was installed on a 3-tier utility cart, the inducing laser (Q-switched Nd:YAG) for LIBS was placed on the middle tier, and the last tier was dedicated for calibration instruments (a NIST traceable balanced Deuterium-Halogen light source for intensity calibration, and some gas/vapor spectral lamps including Mercury-Argon, Argon, Neon, and Krypton for wavelength
\end{abstract}


calibration). The portability feature of this LIBS setup provides a remarkable value for testing and characterizing different biological samples on-site. This is a great capability especially if the target sample has the potential of being contagious. This setup is meant to be used for so-called smart laser osteotomies, i.e., the osteotome will be able to identify the type of the tissue being cut through the feedback provided by LIBS [6$8]$.

Keywords: Spectrometer, Echelle grating, Cross dispenser, LIBS, Tissue characterization

[1] M. Farsad, "A design cycle for echelle spectrometers," Proc. SPIE 10590, 105901F (2017).

[2] M. Hoehse et al.,"A combined laser-induced breakdown and Raman spectroscopy Echelle system for elemental and molecular microanalysis," Spectrochimica Acta Part B: Atomic Spectroscopy 64, 1219-1227 (2009).

[3] M. Sabsabi et al., "Comparative study of two new commercial echelle spectrometers equipped with intensified CCD for analysis of laser-induced breakdown spectroscopy," Applied Optics 42, 6094-6098 (2003).

[4] S. Florek et al., "new, versatile echelle spectrometer relevant to laser induced plasma applications," Spectrochimica Acta Part B 56, 1027-1034 (2001).

[5] C. Haisch, "Combination of an intensified charge coupled device with an echelle spectrograph for analysis of colloidal material by laser-induced plasma spectroscopy," Spectrochimica Acta Part B 53, 1657-1667 (1998).

[6] H. Abbasi et al., "Differentiation of femur bone from surrounding soft tissue using laser induced breakdown spectroscopy as a feedback system for smart laserosteotomy", Proc. SPIE 10685, 1068519 (2018).

[7] F. Mehari et al., "Investigation of the differentiation of ex vivo nerve and fat tissues using laser-induced breakdown spectroscopy (LIBS): Prospects for tissue-specific laser surgery," Journal of Biophotonics 9 (10), 1021-1032 (2016).

[8] R. K. Gill et al., "Preliminary fsLIBS study on bone tumors," Biomedical optics express 6(12), 4850-4858 (2015). 